\title{
Tendencia de la mortalidad por cáncer en niños y adolescentes según grado de marginación en México (1990-2009)
}

\author{
Ricardo Antonio Escamilla-Santiago, MC, M en C,(1) José Narro-Robles, MC,(1) Arturo Fajardo-Gutiérrez, MC, M en C,(2) \\ Ramón Alberto Rascón-Pacheco, MC, M en C, ${ }^{(3)}$ Malaquías López-Cervantes, MC, MPH, PhD.(I)
}

\footnotetext{
Escamilla-Santiago RA, Narro-Robles J, Fajardo-Gutiérrez A, Rascón-Pacheco RA, López-Cervantes M. Tendencia de la mortalidad por cáncer en niños y adolescentes según grado de marginación en México (1990-2009). Salud Publica Mex 20I2;54:587-594.
}

\section{Resumen}

Objetivo. Determinar la tendencia de mortalidad por cáncer en niños y adolescentes por nivel de marginación en México. Material y métodos. La información se obtuvo de certificados de defunción (1990-2009); se calcularon tasas estandarizadas por edad. Se obtuvo el porcentaje promedio anual de cambio (AAPC) calculados con Joinpoint Regression del National Cancer Institute para evaluar la tendencia. Resultados. La mortalidad por cáncer en niños y adolescentes incrementó: en niños fue de $0.87 \%$ hombres y $0.96 \%$ mujeres y $1.22 \%$ hombres y $0.63 \%$ mujeres en adolescentes. El patrón de neoplasias en niños fue leucemias -sistema nervioso- linfomas, y en adolescentes leucemias -huesos y articulacioneslinfomas. Los incrementos de la mortalidad correspondieron a alta y muy alta marginalidad. Conclusión. El incremento de mortalidad correspondiente a los estados de alta y muy alta marginalidad puede explicarse en parte por un mejor registro. Se requieren estudios de sobrevida para evaluar mejor la efectividad de los tratamientos en el país.

Palabras clave: mortalidad infantil; adolescente; neoplasias
Escamilla-Santiago RA, Narro-Robles J, Fajardo-Gutiérrez A, Rascón-Pacheco RA, López-Cervantes M.

Trends of cancer mortality rates in children and adolescents by level of marginalization in Mexico (1990-2009).

Salud Publica Mex 20I2;54:587-594.

\begin{abstract}
Objective. To determine childhood and adolescent cancer mortality by the level of marginalization in Mexico. Materials and methods. We used 1990-2009 death certificates estimating age-standardized rates. We calculated the Average Annual Percent Change (AAPC) using the Joinpoint Regression program available at the National Cancer Institute to assess tendency. Results. Cancer mortality rates increased. AAPC were $0.87 \%$ male and $0.96 \%$ female children, and for adolescents were: males $1.22 \%$ and females $0.63 \%$. The neoplasm pattern in infants was leukemia -central nervous system-lymphomas; and in adolescents it was leukemia -bone and articulation-lymphomas. The increase in cancer mortality corresponded to the high and highest marginated areas of each state. Conclusion. The increase in highly marginated areas may be partly explained by well-documented local registration of deaths. Further studies focusing on survival are required in order to better assess the effectiveness of cancer detection and medical treatment in our country.
\end{abstract}

Key words: infant mortality; adolescent; neoplasms

(I) Facultad de Medicina, Universidad Nacional Autónoma de México. México.

(2) Hospital de Pediatría, CMN Siglo XXI-IMSS. México.

(3) Coordinación de Vigilancia Epidemiológica, IMSS. México.

Fecha de recibido: 2 de mayo de 20II - Fecha de aceptado: 28 de agosto de 2012

Autor de correspondencia: Dr. Malaquías López Cervantes. Unidad de Proyectos Especiales de Investigación, Facultad de Medicina. Torre de investigación, Ier piso, Ciudad Universitaria. 04510 Coyoacán, México, DF, México.

Correo electrónico: mlopezI4@servidor.unam.mx, mlopezcervantes@yahoo.com 
$E^{n}$ el ámbito mundial se ha reportado una tendencia en la disminución de la mortalidad por cáncer en niños. ${ }^{1}$ Además, se considera que particularmente las muertes por cáncer en niños y adolescentes han disminuido en países que cuentan con tecnologías terapéuticas más desarrolladas y lo contrario corresponde a los países en desarrollo que no reflejan esta disminución en sus tasas de mortalidad. ${ }^{2}$

Algunos estudios realizados en Estados Unidos reportan un aumento en la sobrevida de niños con cáncer que han participado en algún protocolo terapéutico. ${ }^{3}$ Las tasas de mortalidad disminuyeron de ocho a menos de tres por cada 100000 niños y la sobrevida aumentó independientemente del tiempo de diagnóstico, a pesar del aumento en la incidencia de la enfermedad. ${ }^{4}$ Distintos países de América, Asia, Europa y Oceanía han presentado un patrón muy similar., ${ }^{5,6}$

En contraste, aún existen países latinoamericanos donde se observa un aumento sostenido de la mortalidad por cáncer en niños y México es uno de los más afectados. ${ }^{5}$ Según cifras del Instituto Nacional de Estadística y Geografía (INEGI), en México el cáncer en niños ocupa los primeros lugares en mortalidad y se encuentra sólo por debajo de las muertes accidentales en el grupo de 1 a 14 años. La gran desigualdad que existe en distintas regiones del país, la falta de acceso a servicios de salud y la pobre tecnología con la que se cuenta pueden ser determinantes en el aumento de las tasas de mortalidad en niños, según se ha reportado en la literatura médica internacional. ${ }^{1}$ Por lo anterior, el objetivo de esta investigación fue determinar si las tendencias de mortalidad por cáncer en niños y adolescentes tienen relación con el nivel de marginación de las entidades federativas de México.

\section{Material y métodos}

Se utilizaron las bases de datos de los certificados de defunción, que son validadas por el INEGI, de los años 1990 a 2009. El grado de marginalidad que se utilizó en el estudio fue de acuerdo con las estimaciones hechas por el Consejo Nacional de Población (Conapo) en 2005.7 También se obtuvieron las proyecciones poblacionales a mitad del año publicadas por el Conapo para el periodo de 1990 a 2009.

Para la selección de los casos de defunción se establecieron los siguientes criterios: a) menores de 20 años: se consideró muerte por cáncer en niños si el sujeto tenía entre 0 a 14 años, y muerte por cáncer en adolescentes si se encontraba entre los 15 y 19 años; $b$ ) nacionalidad mexicana, y c) que el sexo, la edad y la entidad de residencia fueran datos conocidos en los certificados de defunción.
Se realizó un análisis descriptivo que consistió en calcular la razón de la mortalidad entre hombres y mujeres, así como el cálculo de las tasas por edad y sexo. También se estandarizaron las tasas por edad para comparar la mortalidad entre niños y adolescentes.

Adicionalmente, se calculó la mortalidad por tipo de cáncer en diferentes grupos de edad durante el quinquenio de 2005-2009, así como los intervalos de confianza al 95\% para cada uno de los grupos de estudio. Se utilizó la décima versión de la Clasificación Internacional de Enfermedades para identificar los tipos de cáncer, agrupándose en nueve distintos tipos: ${ }^{9}$ huesos y articulaciones (C40-C41); tejidos blandos (C47, C49, C38.0, C45.2); riñón y pelvis renal (C64-C65); cerebro y otros del sistema nervioso (C70, C71, C72); linfoma hodgkin (C81); linfoma no hodgkin (C82-C85, C96.2); leucemias (C91-C95); ojo y órbita (C69) y otros (C00-C37, C39, C43-C44, C46, C48, C50-C63, C66-C68, C73-C80, C $88-C 90$, C97). ${ }^{10}$

El cálculo de las tasas ajustadas por edad se realizó con el método directo y se utilizó la población estándar mundial. ${ }^{11}$ Las tasas se presentan por 100000 habitantes.

Se utilizaron las tasas estandarizadas para el cálculo de la regresión log-lineal de Poisson y posteriormente se determinó la tendencia de la mortalidad utilizando el programa estadístico JointPoint Regression Analysis (JPR). . Este programa utiliza los datos de mortalidad estandarizada durante los periodos de 1990 a 2009 y ajusta un modelo que explique mejor el comportamiento de la tendencia. JPR realiza dos estimaciones: el porcentaje de cambio anual (APC por sus siglas en inglés) y el promedio del porcentaje de cambio anual (AAPC por sus siglas en inglés). El objetivo de este análisis fue encontrar puntos de cambio significativo en la mortalidad detectados en el periodo de estudio. En este análisis se le denomina jointpoint al punto donde el cambio en la tasa de mortalidad es significativo, ya sea en incremento o decremento. Para describir el cambio de tendencia en un periodo se estima el APC o se estima AAPC que pondera los cambios de la tendencia a lo largo del periodo. Se considera que una tendencia es estadísticamente significativa cuando la pendiente es distinta a "cero" con una $p<0.05 .{ }^{12,13}$ Asimismo, para cada entidad federativa se calculó el AAPC según el sexo.

Finalmente, se agruparon las entidades según el grado de marginación para determinar la tendencia de la mortalidad por cáncer en los estados. De acuerdo con

\footnotetext{
* Surveillance Research. Jointpoint Regression Program. Cancer control and population sciences; 2010; Version 3.4.3:[Disponible en: http:// surveillance.cancer.gov/joinpoint/.
} 
el Conapo, se considera que Baja California, Coahuila, Distrito Federal y Nuevo León son de muy baja marginalidad; Aguascalientes, Baja California Sur, Colima, Chihuahua, Jalisco, Estado de México, Morelos, Quintana Roo, Sonora y Tamaulipas son de baja marginalidad; Durango, Guanajuato, Nayarit, Querétaro, Sinaloa, Tlaxcala y Zacatecas son de marginalidad media; Campeche, Hidalgo, Michoacán, Puebla, San Luis Potosí, Tabasco, Veracruz y Yucatán son de alta marginalidad, y Chiapas, Guerrero y Oaxaca pertenecen a un grado de marginalidad muy alto. ${ }^{7}$

\section{Resultados}

Durante el periodo de estudio la mortalidad por cáncer en niños fue de 3.4 a 4.4 y en niñas fue de 2.8 a 3.7 por cada 100000 niños y niñas según se muestra en el cuadro I. El año en el que se encontró la mortalidad más alta en niños fue en 2006, mientras que en las niñas fue durante 2005 y 2007.

En el grupo de adolescentes la mortalidad fue de 6.6 a 8.7 en hombres y 4.6 a 5.9 en mujeres por cada 100000 adolescentes. El año en el que se presentó la mortalidad más alta en los hombres fue 2006 mientras que para las mujeres fue 2004.

En los niños, la razón hombre/ mujer $(\mathrm{H} / \mathrm{M})$ fue de 1.0 a 1.3 y en el caso de los adolescentes entre 1.3 y 1.6.

En relación con la tendencia, sólo en diez estados del país se encontró incremento en la mortalidad por cáncer en niños, por lo que los tres estados con mayor incremento son Guerrero (5.2\%), Chiapas (3.7\%) y Campeche (3.3\%). En el caso de las niñas hubo un incremento en nueve estados, y fue mayor en Durango (3.9\%), Guerrero (2.8\%) y Chiapas (2.6\%) (figura 1$)$.

Entre los adolescentes hombres la mortalidad aumentó en seis estados, por lo que Guerrero (5.7\%), Chihuahua (3.2\%) y Oaxaca (3.1\%) son los que presentaron mayor incremento. En mujeres de este grupo de edad el incremento fue en Baja California (3.9\%), Tamaulipas $(2.9 \%)$ y Chiapas $(2.7 \%)$ (figura 1$)$.

Asimismo, se encontró que la tendencia de la mortalidad aumenta por sexo y grupo de edad, tanto en niños como adolescentes (figura 2).

En forma global la mortalidad por cáncer fue mayor en los adolescentes en comparación de los niños 6.94 (IC 95\% 6.72-7.17) y 4.96 (IC 95\% 4.86-5.08), respectivamente. De acuerdo con el tipo de cáncer, los que causaron mayor mortalidad en niños fueron leucemias 2.61 (IC 95\% 2.54-2.69), del sistema nervioso 0.72 (IC 95\% 0.68-0.77) y linfomas 0.33 (IC 95\% 0.30-0.36). En el caso de los adolescentes fueron leucemias 3.17 (IC 95\% 3.02-3.33), huesos y articulaciones 0.64 (IC 95\% 0.57-0.71) y linfomas 0.62 (IC 0.56-0.69) (cuadro II).

\section{Cuadro I}

MoRTALIDAD POR CÁNCER EN NIÑOS Y ADOLESCENTES MEXICANOS SEGÚN SEXO. 1990-2009

\begin{tabular}{|c|c|c|c|c|c|c|}
\hline \multirow[b]{2}{*}{ Año } & \multicolumn{3}{|c|}{ Cáncer en niños } & \multicolumn{3}{|c|}{ Cáncer en adolescentes } \\
\hline & Hombre* & Mujer* & $\begin{array}{l}\text { Razón } \\
(H / M)\end{array}$ & Hombre ${ }^{\ddagger}$ & Mujer $\neq$ & $\begin{array}{l}\text { Razón } \\
(H / M)\end{array}$ \\
\hline 1990 & 3.7 & 3.4 & 1.1 & 7.0 & 4.7 & 1.5 \\
\hline 1991 & 3.5 & 3.0 & 1.2 & 6.8 & 4.8 & 1.4 \\
\hline 1992 & 3.6 & 2.9 & 1.2 & 7.1 & 4.6 & 1.6 \\
\hline 1993 & 3.8 & 3.0 & 1.3 & 6.6 & 5.1 & 1.3 \\
\hline 1994 & 3.4 & 2.8 & 1.2 & 7.4 & 4.7 & 1.6 \\
\hline 1995 & 3.7 & 3.1 & 1.2 & 7.4 & 5.8 & 1.3 \\
\hline 1996 & 4.0 & 3.5 & 1.1 & 7.2 & 5.6 & 1.3 \\
\hline 1997 & 4.1 & 3.1 & 1.3 & 7.4 & 5.4 & 1.4 \\
\hline 1998 & 3.8 & 3.4 & 1.1 & 8.1 & 5.7 & 1.4 \\
\hline 1999 & 4.0 & 3.4 & 1.2 & 7.8 & 5.2 & 1.5 \\
\hline 2000 & 4.0 & 3.6 & I.I & 7.4 & 4.8 & 1.5 \\
\hline 2001 & 4.1 & 3.3 & 1.2 & 8.3 & 5.3 & 1.6 \\
\hline 2002 & 4.0 & 3.2 & 1.3 & 8.2 & 5.1 & 1.6 \\
\hline 2003 & 4.1 & 3.3 & 1.2 & 8.4 & 5.2 & 1.6 \\
\hline 2004 & 4.2 & 3.5 & 1.2 & 7.8 & 5.9 & 1.3 \\
\hline 2005 & 3.7 & 3.7 & 1.0 & 8.1 & 5.6 & 1.5 \\
\hline 2006 & 4.4 & 3.5 & 1.3 & 8.7 & 5.5 & 1.6 \\
\hline 2007 & 4.1 & 3.7 & 1.1 & 8.5 & 5.3 & 1.6 \\
\hline 2008 & 4.0 & 3.5 & 1.1 & 8.3 & 5.3 & 1.6 \\
\hline 2009 & 4.1 & 3.6 & I.I & 8.4 & 5.7 & 1.5 \\
\hline
\end{tabular}

Los códigos CIE-I0 utilizados para el cálculo de la mortalidad fueron: C00-C99

La mortalidad se calculó por 100000 habitantes

* Tasa ajustada por edad

\# Tasa específica observada

(H) Hombres; (M) Mujeres

Cuando se agruparon las entidades según su grado de marginalidad, se encontró que aumentó la muerte por cáncer en niños de ambos sexos en los grados alto y muy alto, mientras que para los adolescentes incrementó en los hombres y en los grados de marginalidad muy bajo y medio. En el mismo análisis para el grupo de 15 a 19 años, sólo hubo aumento significativo de la mortalidad en los hombres que pertenecían a entidades de media, alta y muy alta marginalidad (figura 3).

\section{Discusión}

En los países desarrollados, durante las décadas recientes se han documentado tres fenómenos relacionados con el cáncer en la niñez y en la adolescencia: la incidencia, que al parecer ha incrementado, una disminución en la mortalidad y un aumento de la sobrevida. ${ }^{2,5,6,14}$ En los Estados Unidos, la mortalidad empezó a disminuir desde 
A

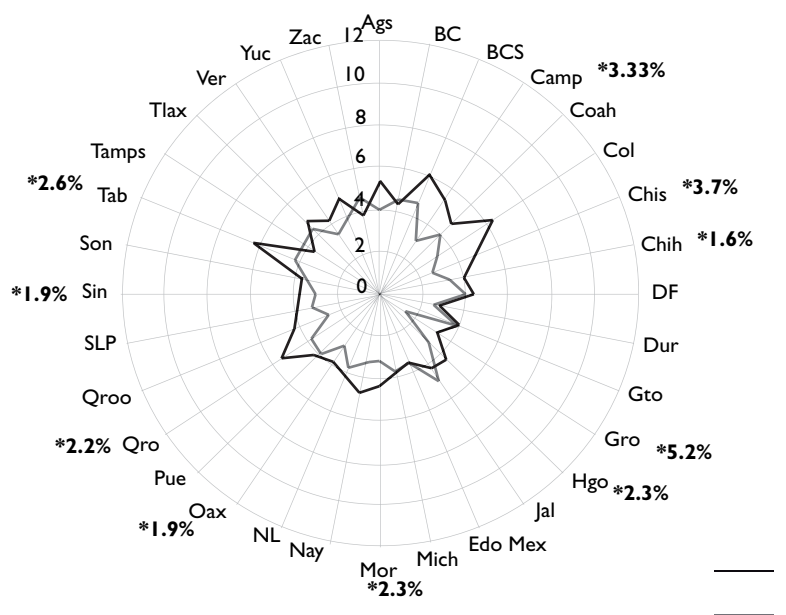

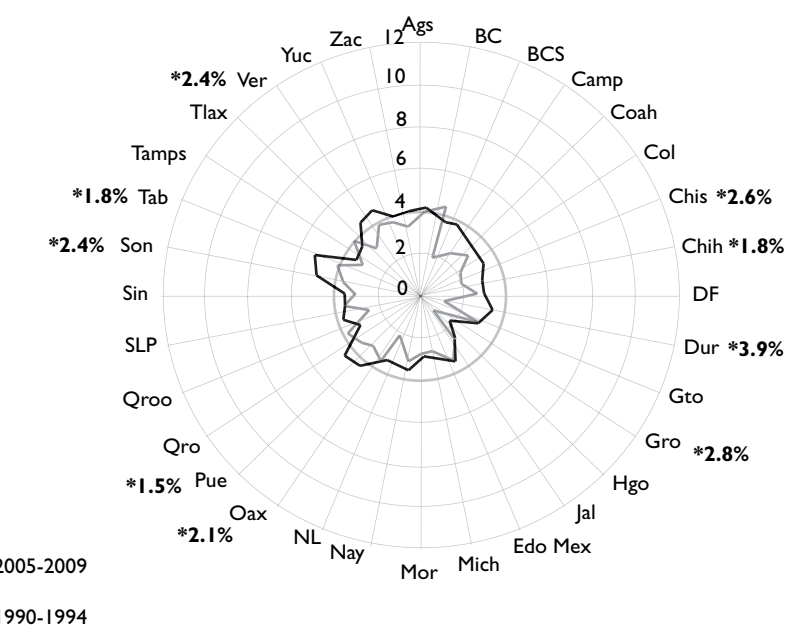

C

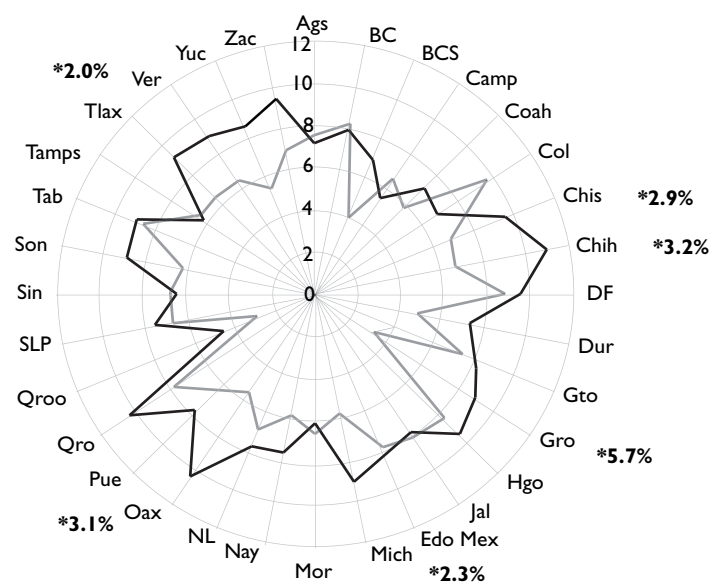

Los códigos CIE-I0 utilizados para el cálculo de la mortalidad fueron: C00-C99 La mortalidad se calculó por 100000 habitantes

A. Mortalidad por cáncer en niños mexicanos B. Mortalidad por cáncer en niñas mexicanas. C. Mortalidad por cáncer en adolescentes hombres mexicanos D. Mortalidad por cáncer en adolescentes mujeres mexicanas

* AAPC durante 1990-2009. ( $p<0.05)$

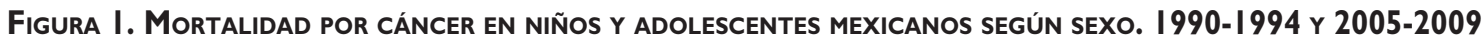

los años sesenta a partir de la creación de instituciones para la atención y apoyo a grupos de niños con cáncer, particularmente del Instituto Nacional del Cáncer. ${ }^{3}$ Dichos esfuerzos se centraron en la formación de personal capacitado y en el establecimiento de protocolos específicos para el tratamiento de dichas enfermedades, donde cada año se registra a más niños con este padecimiento, por lo que las tasas de mortalidad por esta enfermedad han disminuido significativamente. ${ }^{4}$
El estudio de la mortalidad por cáncer en niños tiene gran relevancia porque permite localizar zonas prioritarias donde la gente no está recibiendo tratamiento de manera adecuada o simplemente no tiene oportunidades de acceso a un tratamiento integral. ${ }^{3}$

En México no se tienen suficientes datos para el estudio de la incidencia y la sobrevida debido a que no existe un registro nacional de cáncer, sin embargo, se cuenta con los datos de los certificados de defunción 
Cáncer en niños

9.00

8.00

7.00

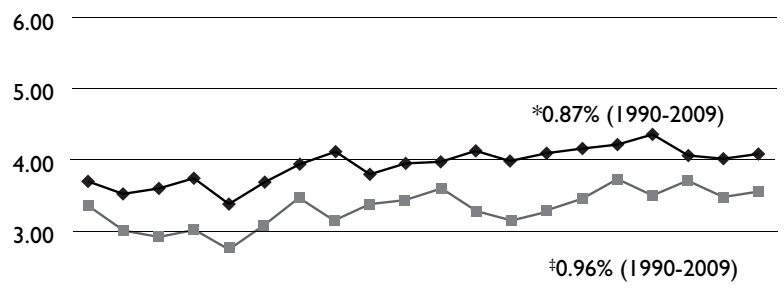

2.00

1.00

0.00

1990

1995
2000

2005
Cáncer en adolescentes

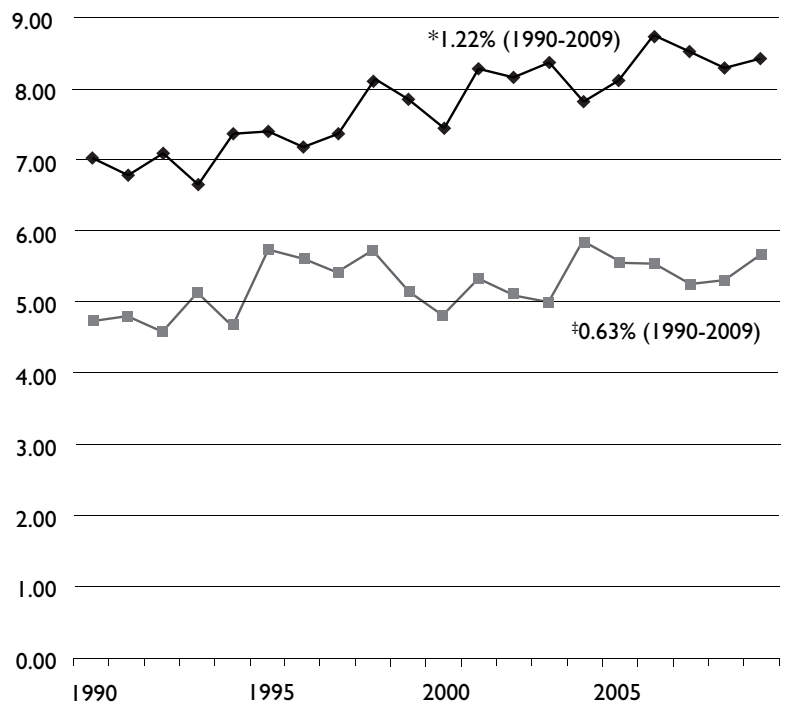

Mujeres

Los códigos CIE-10 utilizados para el cálculo de la mortalidad fueron: C00-C99 La mortalidad se calculó por 100000 habitantes

* AAPC en hombres $p<0.05$

${ }^{\ddagger}$ AAPC en mujeres $p<0.05$

Figura 2. TENDENCIA de LA MORTALIDAD POR CÁNCER EN NIÑOS Y ADOLESCENTES MEXICANOS. 1990-2009

\section{Cuadro II}

MoRTALIDAd POR TIPO DE CÁNCER EN NIÑOS Y ADOLESCENTES MEXICANOS. 2005-2009

\begin{tabular}{|c|c|c|c|c|c|c|c|c|}
\hline \multirow{2}{*}{$\begin{array}{l}\text { Tipos de cáncer } \\
\text { Edad (años) }\end{array}$} & \multicolumn{4}{|c|}{ Cáncer en niños } & \multicolumn{4}{|c|}{ Cáncer en adolescentes } \\
\hline & $<1$ & l a 4 & 5 a9 & 10 a 14 años & $0 a / 4$ & IC $95 \%$ & 15 a 19 & IC $95 \%$ \\
\hline & años & años & años & años & años & & años & \\
\hline Huesos y articulaciones $*(\mathrm{C} 40-\mathrm{C} 4 \mathrm{I})$ & 0.03 & 0.05 & 0.14 & 0.42 & 0.21 & $(0.19-0.23)$ & 0.64 & $(0.57-0.7 \mathrm{I})$ \\
\hline Tejidos blandos *(C47,C49,C38, C45.2) & 0.14 & 0.22 & 0.17 & 0.17 & 0.18 & $(0.16-0.20)$ & 0.31 & $(0.27-0.36)$ \\
\hline Riñón y pelvis renal *(C64-C65) & 0.17 & 0.26 & 0.14 & 0.05 & 0.14 & $(0.13-0.16)$ & 0.06 & $(0.04-0.09)$ \\
\hline Sistema nervioso $*(\mathrm{C} 70, \mathrm{C7I}, \mathrm{C} 72)$ & 0.44 & 0.78 & 0.89 & 0.57 & 0.72 & $(0.68-0.77)$ & 0.53 & $(0.47-0.60)$ \\
\hline Linfomas *(C8I-C85, C96.2) & 0.09 & 0.27 & 0.34 & 0.39 & 0.33 & $(0.30-0.36)$ & 0.62 & $(0.56-0.69)$ \\
\hline Leucemias *(C9I-C95) & 1.79 & 2.75 & 2.61 & 2.67 & 2.61 & $(2.54-2.69)$ & 3.17 & $(3.02-3.33)$ \\
\hline Ojo y órbita *(C69) & 0.05 & 0.32 & 0.06 & 0.01 & 0.11 & $(0.10-0.13)$ & 0.02 & $(0.01-0.04)$ \\
\hline Otros $^{\ddagger}$ & 1.05 & 0.99 & 0.45 & 0.56 & 0.66 & $(0.62-0.70)$ & 1.59 & $(1.48-1.70)$ \\
\hline Total & 3.76 & 5.64 & 4.80 & 4.84 & 4.96 & $(4.86-5.08)$ & 6.94 & $(6.72-7.17)$ \\
\hline
\end{tabular}

La mortalidad se calculó por 100000 habitantes

* Los código CIE-10 utilizados para cada tipo de cáncer

¥ Los códigos ClE-10 utilizados (C00-C37; C39; C43-C44; C46; C48; C50-C63; C66-C68; C73-C80; C88-C90: C97) 
A. Mortalidad por cáncer en niños ( 0 a 14 años) según sexo y grado de marginalidad
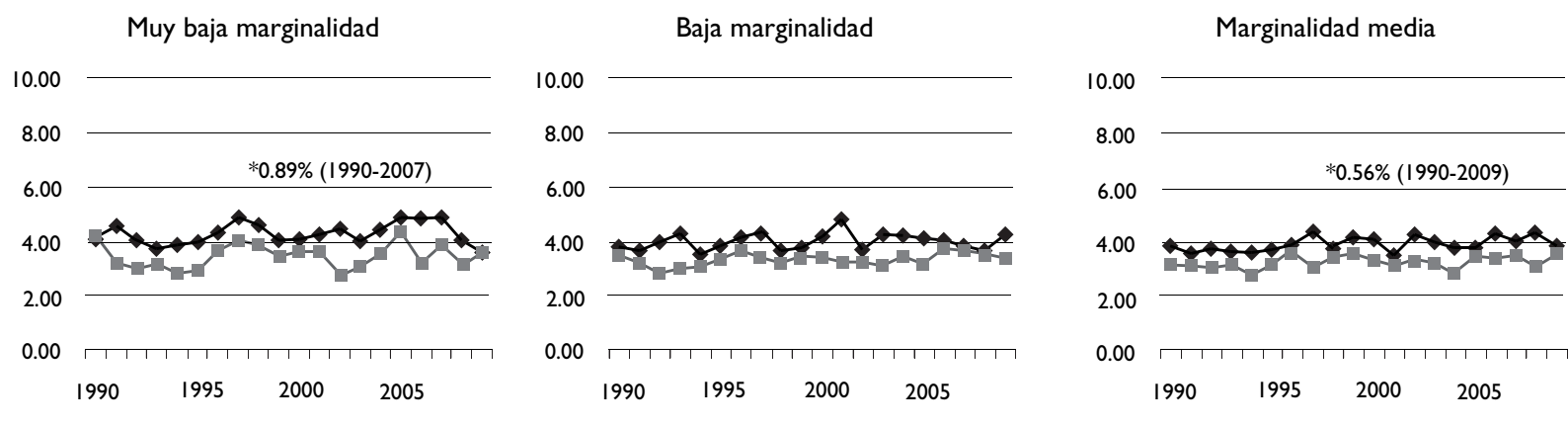

Alta marginalidad

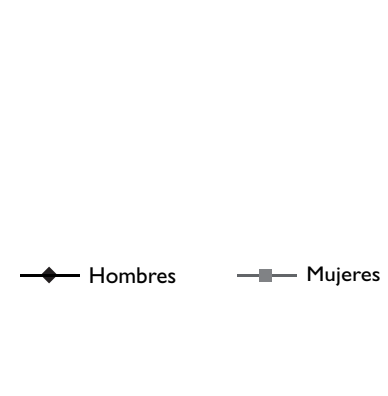

Alta marginalidad
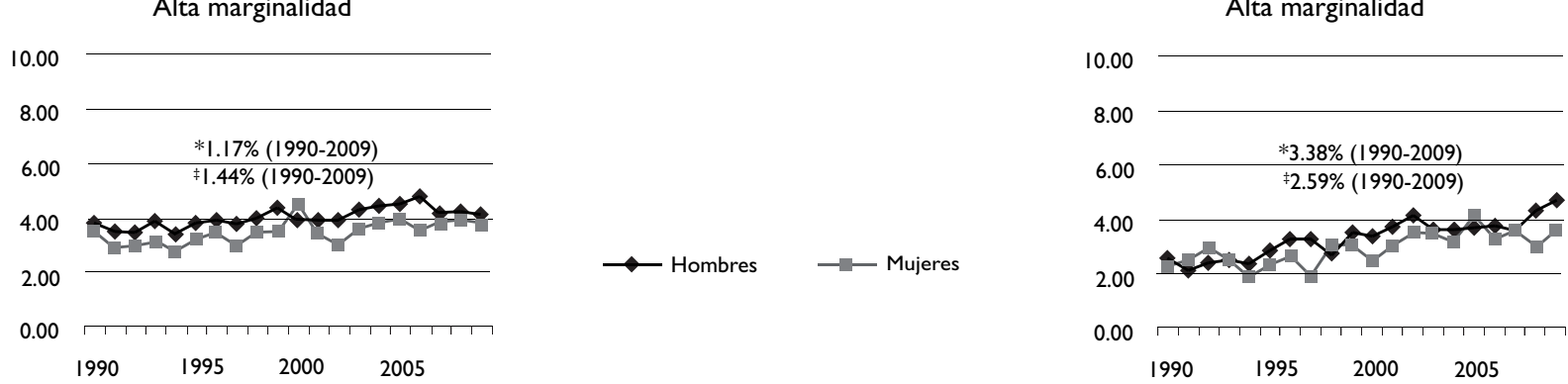

B. Mortalidad por cáncer en adolescentes (15 a 19 años) según sexo y grado de marginalidad

Muy baja marginalidad

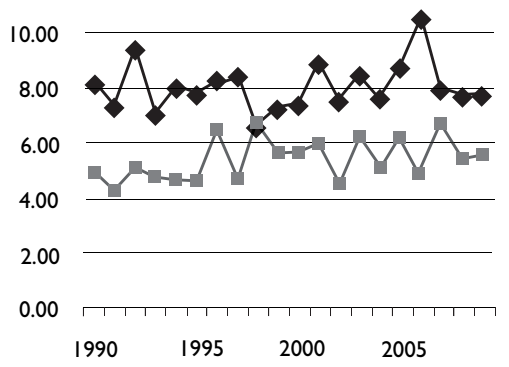

Alta marginalidad

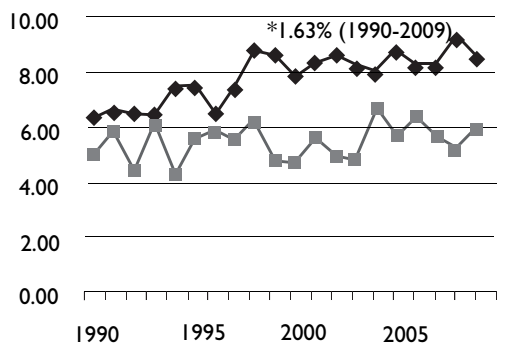

Baja marginalidad

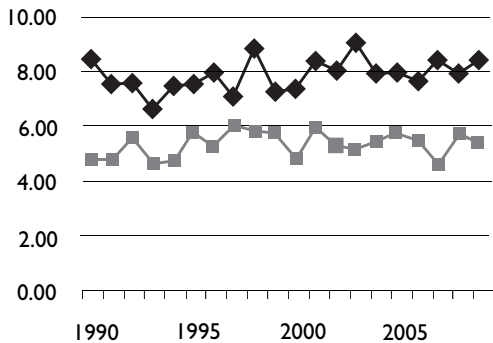

$\longrightarrow$ Hombres $\rightarrow$ Mujeres
Marginalidad media

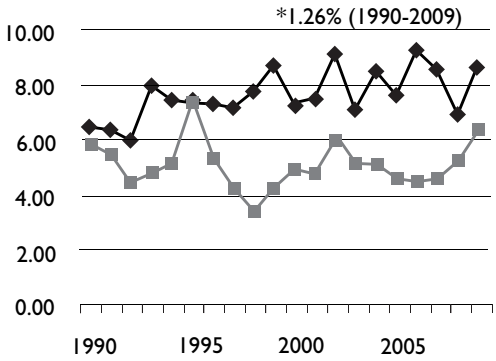

Alta marginalidad

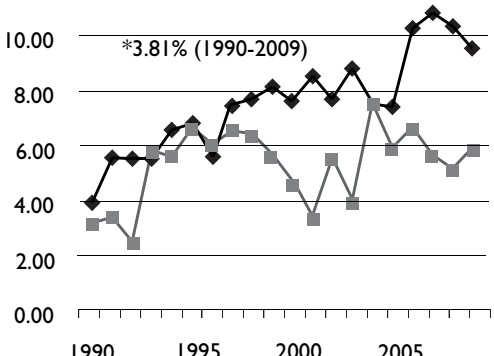

Los códigos CIE-10 utilizados para el cálculo de la mortalidad fueron: C00-C99

La mortalidad se calculó por 100000 habitantes

* AAPC en hombres $p<0.05$

$\ddagger$ AAPC en mujeres $p<0.05$

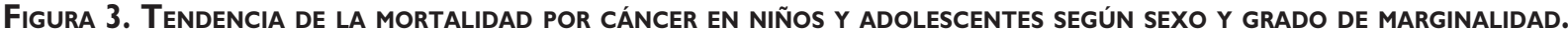
1990-2009 
que resultaron de gran utilidad para identificar un incremento en las tasas de mortalidad por cáncer en niños y adolescentes en los últimos 20 años; el mayor aumento es en el caso de los adolescentes. Cabe aclarar que las diferencias en las tasas de mortalidad ajustadas con otros estudios recientes pueden ser debidas al uso de distintos denominadores. ${ }^{2,5}$

El patrón de mortalidad que presentaron los niños con cáncer en México fue el estadounidense/ europeo ${ }^{15}$ que presenta mayor número de muertes por leucemias, seguidas por tumores del sistema nervioso y linfomas. Por otro lado, en los adolescentes predominaron las leucemias, seguidas del cáncer de huesos y articulaciones y linfomas. Una de las limitaciones del uso de los certificados de defunción en estos estudios es que registran información que se clasifica de manera topográfica y no morfológica como en el caso de la clasificación internacional de cáncer infantill, ${ }^{16}$ por lo que los resultados que se interpretaron pueden tener limitaciones. Algunos de los tipos de cáncer se categorizaron como no clasificados por lo que es difícil conocer el impacto en la mortalidad infantil de estos casos.

La tendencia de la mortalidad por cáncer en niños y adolescentes en México aumentó, pero el incremento del AAPC en los últimos 20 años fue más evidente en estados de muy alta marginalidad tanto en niños como en adolescentes.

En un estudio realizado en países europeos se observó que en el año de 1990 México compartía la misma tasa de mortalidad con otros 20 países. Para 2007, México no sólo presentaba un aumento en la tendencia de la mortalidad junto con Estonia y Latvia, sino que presentó la mayor tasa de mortalidad por cáncer en niños. ${ }^{3}$ En otro estudio donde a México se le compara con países de América, Asia y Oceanía se reportan los mismos resultados que el estudio europeo. ${ }^{5}$

El estudio de la mortalidad por cáncer en niños en países donde no se tienen registros de cáncer de base poblacional traduce la efectividad en los tratamientos y el acceso a ellos, por lo que un incremento en las tasas de mortalidad hace evidente un mal manejo terapéutico y poco acceso de estas opciones para los niños que padecen esta enfermedad.

Cuando se estudian tendencias de la mortalidad es conveniente descartar que los resultados sean debidos a tres aspectos: un cambio en la clasificación de la enfermedad, el uso de nuevas tecnologías para el diagnóstico y un mejor registro de las muertes. ${ }^{17}$

En este estudio se observó un incremento en la tendencia de la mortalidad por cáncer en estados con alta y muy alta marginalidad, lo que puede traducirse que en dichos estados se haya mejorado el registro de muertes por cáncer porque los otros dos aspectos no se han implementado en México. Independientemente de cuál sea la causa de esta tendencia, lo cierto es que en el país no se observa un descenso en la mortalidad por cáncer en menores de 20 años por lo que surgen los siguientes retos. Por un lado, crear un registro nacional de cáncer para que a partir de él se realice un mayor número de investigaciones donde se estudie el verdadero comportamiento de la incidencia y la sobrevida de niños con cáncer en México. Lo anterior permitiría poder evaluar los resultados efectivos en relación con el tratamiento del cáncer en México con respecto al resto del mundo, donde se observa un aumento claro en la sobrevida. ${ }^{4}$

Otro tema importante que se debe analizar es el estudio del cáncer en adolescentes y las instituciones de salud donde son atendidos; ya existen estudios que indican que la atención es mejor en centros pediátricos que en los de adultos. ${ }^{18}$

Puede concluirse que en este estudio se encontró una mortalidad por cáncer, tanto en niños como en adolescentes, mayor a la reportada en países desarrollados como Estados Unidos de América, ${ }_{10}$ así como una tendencia al incremento en estados con alta y muy alta marginalidad, lo cual posiblemente se debe a un mejor registro de los datos de mortalidad.

Declaración de conflicto de intereses: Los autores declararon no tener conflicto de intereses.

\section{Referencias}

I. Schottenfeld D, Fraumeni JF. Cancer epidemiology and prevention. 3rd ed. Oxford; New York: Oxford University Press, 2006.

2. La Vecchia C, Levi F, Lucchini F, Lagiou P, Trichopoulos D, Negri E. Trends in childhood cancer mortality as indicators of the quality of medical care in the developed world. Cancer 1998;83(10):2223-2227.

3. Bleyer WA. The US pediatric cancer clinical trials programmes: international implications and the way forward [Internet]. Eur J Cancer 1997;33(9):I439- 1447. Available from: http://linkinghub.elsevier.com/ retrieve/pii/S0959804997002499

4. Bleyer WA. The impact of childhood cancer on the United States and the world. [Internet]. CA a cancer journal for clinicians 1990;40(6):355367. Available from: http://doi.wiley.com/ $10.3322 /$ canjclin.40.6.355 5. Chatenoud L, Bertuccio P, Bosetti C, Levi F, Negri E, La Vecchia C. Childhood cancer mortality in America, Asia, and Oceania, 1970 through 2007 [Internet]. Cancer 2010; I 6(2I):5063-5074. Available from: http:// www.ncbi.nlm.nih.gov/pubmed/20629033

6. Bosetti C, Bertuccio P, Chatenoud L, Negri E, Levi F, La Vecchia C. Childhood cancer mortality in Europe, 1970-2007 [Internet]. Eur J Cancer 2010;46(2):384-394. Available from: http://www.ncbi.nlm.nih.gov/ pubmed/I9818600

7. Carlos Anzaldo, MP. Índices de Marginación 2005. In: Consejo Nacional de Población. México: Conapo, 2005.

8. Betty Kikwood JS. Medical Statistics. Second edition ed. Massachusetts, USA: Blackwell Science Ltd, 2003. 
9. Organización Panamericana de la Salud. Clasificación estadística internacional de enfermedades y problemas relacionados con la salud. Washington, DC: OPS, 1995.

10. US. Cancer Statistics Working Group. United States Cancer Statistics: $200 I$ Incidence and Mortality. In: Services DoHaH, ed. US: Center for Disease Control and Prevention and National Cancer Institute, 2004. II. Waterhouse J. Cancer incidence in five continents. Lyon: IARC, 1976 12. Kim H-J, Fay MP, Yu B, Barrett MJ, Feuer EJ. Comparability of segmented line regression models [Internet]. Biometrics 2004;60(4): 10051014. Available from: http://www.ncbi.nlm.nih.gov/pubmed/I560642 I 13. Kim HJ, Fay MP, Feuer EJ, Midthune DN. Permutation tests for joinpoint regression with applications to cancer rates [Internet]. Statistics in Medicine 2000; 19(3):335-35I. Available from: http://www.ncbi.nlm.nih. gov/pubmed/10649300

14. Kaatsch P. Epidemiology of childhood cáncer. Cancer Treat Rev 2010;36(4):277-285.
15. Fajardo-Gutiérrez A, Juárez-Ocaña S, González-Miranda G, PalmaPadilla V, Carreón-Cruz R, Ortega-Álvarez MC, et al. Incidence of cancer in children residing in ten jurisdictions of the Mexican Republic: Importance of the Cancer Registry (A population.based study). BMC Cancer 2007,7:68. Available from: http://www.biomedcentral.com//47I $2407 / 7 / 68$

16. Steliarova-Foucher E, Stiller C, Lacour B, Kaatsch P. International Classification of Childhood Cancer. 3er ed [Internet]. Cancer 2005; I03(7):I457- I 467. Available from: http://www.ncbi.nlm.nih.gov/ pubmed/157/2273

17. Moriyama IM. Preliminary observations on recent mortality trend. Public health reports Washington DC 1974;76(I2): 1056-I058.

18. Desandes E. Survival from adolescent cancer [Internet]. Cancer Treatment Reviews 2007;33(7):609-615. Available from: http://www.ncbi. nlm.nih.gov/pubmed/I73980II 